PROCEEDINGS OF THE

AMERICAN MATHEMATICAL SOCIETY

Volume 33, Number 1, May 1972

\title{
ON TORSION IN $H$-SPACES OF RANK TWO
}

KAI K. DAI

Abstract. Let $X$ be an arcwise connected $H$-space that is also a finite $C W$ complex. If $X$ has rank two and if its mod 2 cohomology is primitively generated, then $X$ has no $p$-torsion for $p \geqq 5$.

Let $X$ be an arcwise connected $H$-space that is also a finite CW complex. A classical theorem of Hopf says that the reduced cohomology of $X$ with rational coefficients is an exterior algebra on odd dimensional generators. If it is generated by $x_{1}, \cdots, x_{n}$ in dimensions $d_{1}, \cdots, d_{n}$, respectively, with $d_{i} \leqq d_{i+1}$, then $X$ is said to have rank $n$ and have type $\left(d_{1}, \cdots, d_{n}\right)$. If $H^{i}(X ; Z)$ contains an element of order $p$ for some $i$ and some prime $p$, then $X$ is said to have $p$-torsion.

In $\S 6$ of [8] it is shown that if $X$ is an arcwise connected $H$-space that is also a finite $\mathrm{CW}$ complex and if $X$ is of rank one, then $X$ has no $p$-torsion for all odd primes $p$. The purpose of this note is to show:

THEOREM. Let $X$ be an arcwise connected $H$-space that is also a finite $\mathrm{CW}$ complex. If $X$ has rank two and if its mod 2 cohomology is primitively generated, then $X$ has no $p$-torsion for $p \geqq 5$.

Proof. We divide into two cases, namely: (i) $X$ has no 2-torsion, and (ii) $X$ has 2-torsion.

(i) If $X$ has no 2-torsion, then the type of $X$ is $(1,1),(1,3),(1,7),(3,3)$, $(3,5),(3,7)$, or $(7,7)$. We remark here that this result was partially proved by J. Adams [3] and completed by Douglas-Sigrist [9] and was also proved independently by J. Hubbuck [10, Theorem 1.1]. Suppose that $X$ has $p$-torsion for $p \geqq 5$. If $s$ is the smallest integer for which $H^{s}(X ; Z)$ has $p$-torsion, then, by [7, Theorem 6.4], $s=2 m$ and. since $p \neq 2, H_{2 m}\left(X ; Z_{p}\right)$ has a primitive element. Since $P\left(H_{2 m}\left(X ; Z_{p}\right)\right) \cong\left(Q\left(H^{2 m}\left(X ; Z_{p}\right)\right)^{*}\right.$ [11], where $P\left(H_{2 m}\left(X ; Z_{p}\right)\right)$ is the subspace of primitive elements of $H_{2, n}\left(X ; Z_{p}\right)$, and $\left(Q\left(H^{2 m}\left(X ; Z_{p}\right)\right) *\right.$ is the dual of the subspace of indecomposable elements of $H^{2 m}\left(X ; Z_{p}\right)$, we see that $H^{2 m}\left(X ; Z_{p}\right)$ has an indecomposable element; hence $H^{2 m}\left(X ; Z_{p}\right)$ has a generator. By [7, Theorem 4.7], $H^{*}(X ; Q)$ has a generator in dimension $2 m p^{k}-1$, for some $k, 0<k<\infty$.

Received by the editors April 28, 1971 and, in revised form, July 12, 1971.

AMS 1970 subject classifications. Primary 57F25.

$K e y$ words and phrases. $H$-spaces, rank, type, $p$-torsion, primitive and indecomposable elements. 
We have $2 m \geqq 2, p \geqq 5$, and $k \geqq 1$; so $2 m p^{k}-1 \geqq 9$. This contradicts the possible types of $X$ which are $(1,1),(1,3),(1,7),(3,3),(3,5),(3,7)$ and $(7,7)$.

(ii) If $X$ has 2-torsion, then again we consider two cases: (a) $X$ is simply connected, and (b) $X$ is not simply connected.

(a) If $X$ has 2-torsion and is simply connected, then we have:

$$
H^{*}\left(X ; Z_{2}\right) \cong H^{*}\left(G_{2} ; Z_{2}\right),
$$

where the exceptional Lie group $G_{2}$ has mod 2 cohomology [4]:

$$
H^{*}\left(G_{2} ; Z_{2}\right) \cong Z_{2}\left[x_{3}\right] /\left(x_{3}^{4}\right) \otimes \wedge\left(S q^{2} x_{3}\right)
$$

the tensor product of a polynomial algebra on one generator of dimension 3 truncated at height 4 and an exterior algebra on one generator of dimension 5. Since $H^{14}\left(G_{2} ; Z_{2}\right) \neq 0$ and $H^{i}\left(G_{2} ; Z_{2}\right)=0$ for $i>14$, we have $H^{14}\left(X ; Z_{2}\right) \neq 0$ and $H^{i}\left(X ; Z_{2}\right)=0$ for $i>14$. By [6, Theorem 7.1] we have $H^{14}(X ; Q) \neq 0$ and $H^{i}(X ; Q)=0$ for $i>14$. Now if $X$ has $p$-torsion for $p \geqq 5$, then by the argument used in (i) above we see that there is a generator for $H^{*}(X ; Q)$ in dimension $2 m p^{k}-1$, where $2 m$ is the smallest integer for which $H^{2 m}(X ; Z)$ has $p$-torsion. Since $X$ is simply connected, we have $\pi_{1}(X)=\pi_{2}(X)=0$ by [6, Theorem 6.11]. This implies that $H^{1}(X)=H^{2}(X)=0$. Thus $2 m \geqq 4, p \geqq 5, k \geqq 1$, and so $2 m p^{k}-1 \geqq 19$. This contradicts the fact that $H^{i}(X ; Q)=0$ for $i>14$.

(b) If $X$ has 2-torsion and is not simply connected, then consider the universal covering space $\tilde{X}$ of $X$. First, observe that $X$ does not have type $(1,1)$. If $X$ has type $(1,1)$, then $H^{2}(X ; Z)=Z$ and $H^{i}(X ; Z)=0$ for $i>2$ [6, Corollary 7.2]. Since $X$ has 2-torsion, $H^{2 n}(X ; Z)$ contains an element of order 2, where $2 n$ is the smallest integer for which $H^{2 n}(X ; Z)$ has 2torsion. Since $2 n \geqq 2$, we have a contradiction; hence $X$ does not have type $(1,1)$. Also, notice that $\tilde{X}$ is a simply connected $H$-space and that $X$ is of the homotopy type of a finite $\mathrm{CW}$ complex [12]. From [5] we have that if $\left(d_{1}, \cdots, d_{n}\right)$ is the type of $\tilde{X}$, then the possible types of $X$ are $\left(d_{1}, \cdots, d_{n}\right)$ and $\left(1,1, \cdots, 1, d_{1}, \cdots, d_{n}\right)$. Let $\tilde{X}$ have type $\left(d_{1}\right)$, then by [1] or [8] we have $d_{1}=3$ or 7 . This implies that $X$ has type $(1,3)$ or $(1,7)$, in which case there is no $p$-torsion for $p \geqq 5$ by the argument used in (i) above. Now, let $\tilde{X}$ have rank two. From (i) above, we see that if $\tilde{X}$ has no 2-torsion, then $\tilde{X}$ has type $(3,3),(3,5),(3,7)$, or $(7,7)$. So the type of $X$ is $(3,3)$, $(3,5),(3,7)$, or $(7,7)$. As reasoned in (i) above, $X$ has no $p$-torsion for $p \geqq 5$ when $\tilde{X}$ has no 2-torsion. If $\tilde{X}$ has 2 -torsion, then again we have:

$$
H^{*}\left(\tilde{X} ; Z_{2}\right) \cong H^{*}\left(G_{\varepsilon} ; Z_{2}\right) \text {. }
$$

$X$ has no $p$-torsion for $p \geqq 7$ because if it does, $H^{*}(X ; Q)$ will have a generator in a dimension at least $2(1) 7-1=13$. This implies that the type 
of $X$ is $(1,13)$ since the top dimension of $H^{*}(X ; Q)$ is 14 . But then $\tilde{X}$ is of rank one, a contradiction to our hypothesis that $\tilde{X}$ is of rank two. Suppose $X$ has 5-torsion. Then $H^{*}(X ; Q)$ has a generator in dimension $2 m 5^{k}-1$ for $m \geqq 1, k \geqq 1$, where $2 m$ is the smallest integer for which $H^{2 m}(X ; Z)$ has 5 -torsion. The only dimension to consider is 9 since the top dimension is 14. Again this implies that the type of $X$ is $(5,9)$. Now, $X$ has 2-torsion, so let $2 n$ be the smallest dimension in which $H^{*}(X ; Z)$ has 2-torsion. We see that $2 n$ is not 2,4 , or 8 since then $H^{*}(X ; Q)$ will have a generator in dimension $2(1) 2^{k}-1,2(2) 2^{k}-1$, or $2(4) 2^{k}-1$, respectively, which is not 5 or 9 . Thus 6 and 10 are the only remaining possibilities. In either case $H^{3}\left(X ; Z_{2}\right)=H^{1}\left(X ; Z_{2}\right)=0$. But $H^{3}\left(\tilde{X} ; Z_{2}\right) \cong H^{3}\left(G_{2} ; Z_{2}\right) \cong Z_{2}$ so by [5] we have a contradiction. This completes the proof of the theorem.

REMARK. The theorem above says that $X$ has no $p$-torsion for $p \geqq 5$. In fact, $X$ may have 2-torsion; simply let $X=G_{\because .}$. Also, $X$ may have 3torsion. Consider $S U(3)$. Its center is $Z_{3}$ [2]. Let $X$ be the corresponding projective group $P S U(3)$. It is obvious that $H^{2}(X ; Z) \cong Z_{3}$.

\section{REFERENCES}

1. J. F. Adams, On the non-existence of elements of Hopf invariant one, Ann. of Math. (2) 72 (1960), 20-104. MR 25 \#530.

2. - Lectures on Lie groups, Benjamin. New York, 1969. MR 40 \#5780.

3. - H-spaces with few cells, Topology 1 (1962), 67-72. MR 26 \#5574.

4. A. Borel, Sur l'homologie et la cohomologie des groupes de Lie compacts connexes, Amer. J. Math. 76 (1954), 273-342. MR 16, 219.

5. W. Browder, The cohomology of covering spaces of $H$-spaces, Bull. Amer. Math. Soc. 65 (1959), 140-141. MR 22 \#1891.

6. - Torsion in H-spaces, Ann. of Math. (2) 74 (1961), 24-51. MR 23 \#A2201.

7. - On differential Hopf algebras, Trans. Amer. Math. Soc. 107 (1963), 153176. MR $26 \# 3061$.

8. _- Higher torsion in H-spaces, Trans. Amer. Math. Soc. 108 (1963), 353-375. MR 27 \#5260.

9. R. Douglas and F. Sigrist, Sphere bundles over spheres and $H$-spaces, Topology 8 (1969), 115-118. MR 39 \#6312.

10. J. R. Hubbisck, Generalized cohomology operations and H-spaces of low rank, Trans. Amer. Math. Soc. 141 (1969), 335-360. MR 40 \#2059.

11. J. W. Milnor and J. C. Moore, On the structure of Hopf algebras, Ann. of Math. (2) 81 (1965), 211-264. MR 30 \#4259.

12. J.-P. Serre, Homologie singulière des espaces fibrés. Applications, Ann. of Math. (2) 54 (1951), 425-505. MR 13, 574.

Department of Mathematics, Michigan State University, East Lansing, Michigan 48823 (Current address)

Department of Mathematics, Dartmouth College, Hanover, New Hampshire 03755 